\title{
Routes to covalent catalysis by reactive selection for nascent protein nucleophiles
}

Dr. Andrey V. Reshetnyak, PhD

Ms. Maria Francesca Armentano

Dr. Natalia A. Ponomarenko, PhD

Ms. Domenica Vizzuso

Ms. Oxana M. Durova

Mr. Rustam Ziganshin

Dr. Marina Serebryakova, $P h D$

Dr. Vadim Govorun, PhD

Dr. Gennady Gololobov, PhD

Dr. Herbert C. Morse III, PhD

Professor Alain Friboulet, PhD

Professor Sudesh P. Makker, MD

Professor Alexander G. Gabibov, PhD

Professor Alfonso Tramontano, PhD 

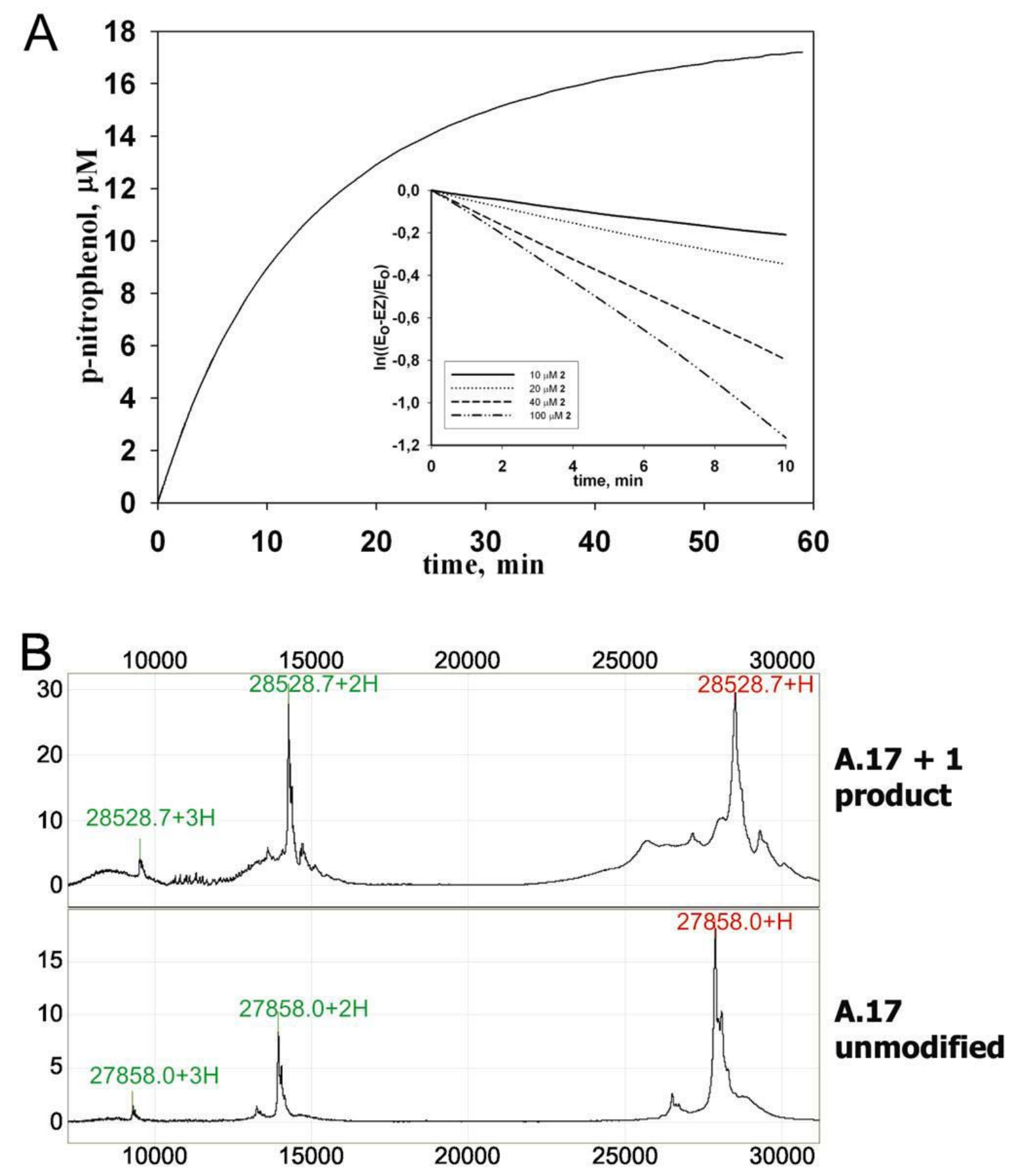

Figure S1. Stoichiometry of scFv phosphonylation. A. Active center titration of a typical reaction of A.17 $(18 \mu \mathrm{M})$ with $100 \mu \mathrm{M}$ of $\mathbf{2}$. The insert shows kinetics of $\mathbf{A . 1 7}$ modification by $\mathbf{2}$ as determined by the Kitz-Willson equation. B. SELDI MS analysis of A.17 $* \mathbf{1}$ reaction product and unmodified A.17. The mass difference between labeled and unmodified scFv corresponded to one phosphonate residue. 


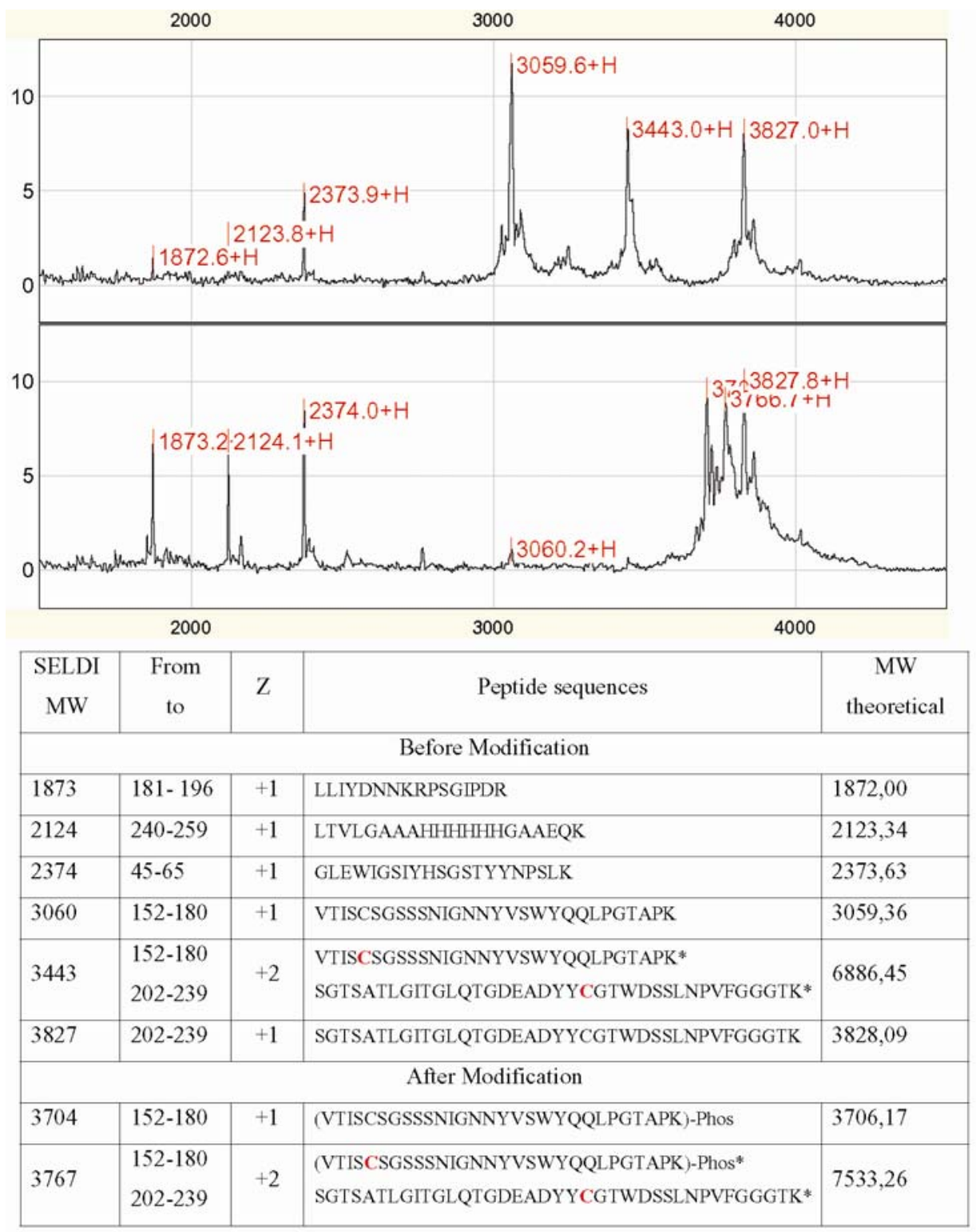

Figure S2. SELDI MS analysis of tryptic hydrolysate of A.17 (upper spectrum) and A.17 labeled with 1 (lower spectrum). Peptide sequences and theoretical and experimental molecular masses are presented in the table. The peptide sequences are identified according to $\mathrm{scFv}$ sequence numbering. Peptide 152 - 180 corresponded to CDR-L1 and flanking residues from FR-L1 and FR-L2 regions (VL 18-45 according to Kabat numbering). 

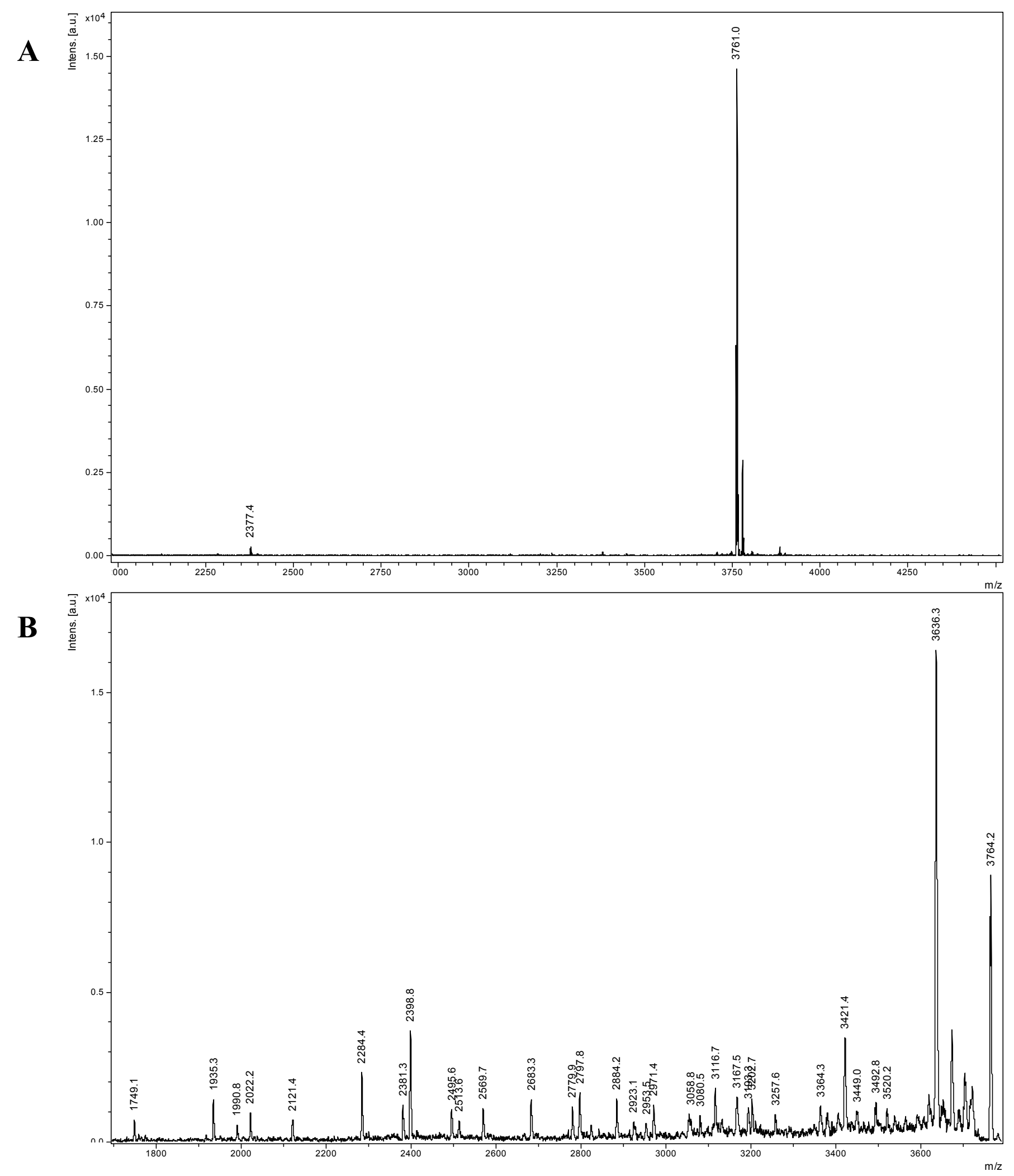
C.

\begin{tabular}{|c|c|c|c|c|c|c|c|c|}
\hline a & $\mathrm{b}$ & $C^{\prime} '$ & \multicolumn{3}{|c|}{ Res: } & $\mathrm{x}$ & $y^{\prime} '$ & $\mathrm{Z}$ \\
\hline 72,081 & 100,076 & 151,108 & 1 & $\mathrm{Val}$ & 29 & - & - & - \\
\hline 173,129 & 201,124 & 252,156 & 2 & Thr & 28 & 3688,709 & 3662,730 & 3643,688 \\
\hline 286,213 & 314,208 & 365,240 & 3 & Ile & 27 & 3587,662 & 3561,683 & 3542,640 \\
\hline 373,245 & 401,240 & 452,272 & 4 & Ser & 26 & 3474,578 & 3448,599 & 3429,556 \\
\hline 533,276 & 561,271 & 612,303 & 5 & Cys & 25 & 3387,546 & 3361,567 & 3342,524 \\
\hline 620,308 & 648,303 & 699,335 & 6 & Ser & 24 & 3227,515 & 3201,536 & 3182,494 \\
\hline 677,329 & 705,324 & 756,356 & 7 & Gly & 23 & 3140,483 & 3114,504 & 3095,462 \\
\hline 764,361 & 792,356 & 843,388 & 8 & Ser & 22 & 3083,462 & 3057,482 & 3038,440 \\
\hline 851,393 & 879,388 & 930,420 & 9 & Ser & 21 & 2996,429 & 2970,450 & 2951,408 \\
\hline 938,425 & 966,420 & 1017,452 & 10 & Ser & 20 & 2909,397 & 2883,418 & 2864,376 \\
\hline 1052,468 & 1080,463 & 1131,495 & 11 & Asn & 19 & 2822,365 & 2796,386 & 2777,344 \\
\hline 1165,552 & 1193,547 & 1244,579 & 12 & Ile & 18 & 2708,323 & 2682,343 & 2663,301 \\
\hline 1222,574 & 1250,569 & 1301,601 & 13 & Gly & 17 & 2595,238 & 2569,259 & 2550,217 \\
\hline 1336,617 & 1364,612 & 1415,644 & 14 & Asn & 16 & 2538,217 & 2512,238 & 2493,196 \\
\hline 1450,660 & 1478,655 & 1529,687 & 15 & Asn & 15 & 2424,174 & 2398,195 & 2379,153 \\
\hline 1613,723 & 1641,718 & 1692,750 & 16 & Tyr & 14 & 2310,131 & 2284,152 & 2265,110 \\
\hline 1712,791 & 1740,786 & 1791,818 & 17 & Val & 13 & 2147,068 & 2121,089 & 2102,046 \\
\hline 1799,823 & 1827,818 & 1878,850 & 18 & Ser & 12 & 2047,999 & 2022,020 & 2002,978 \\
\hline 1985,903 & 2013,898 & 2064,930 & 19 & $\operatorname{Trp}$ & 11 & 1960,967 & 1934,988 & 1915,946 \\
\hline 2795,285 & 2823,280 & 2874,312 & 20 & Tyr & 10 & 1774,888 & 1748,909 & 1729,867 \\
\hline 2923,344 & 2951,339 & 3002,371 & 21 & Gln & 9 & 965,506 & 939,526 & 920,484 \\
\hline 3051,402 & 3079,397 & 3130,429 & 22 & Gln & 8 & 837,447 & 811,468 & 792,425 \\
\hline 3164,486 & 3192,481 & 3243,514 & 23 & Leu & 7 & 709,388 & 683,409 & 664,367 \\
\hline 3261,539 & 3289,534 & 3340,566 & 24 & Pro & 6 & 596,304 & 570,325 & 551,283 \\
\hline 3318,561 & 3346,556 & 3397,588 & 25 & Gly & 5 & 499,252 & 473,272 & 454,230 \\
\hline 3419,608 & 3447,603 & 3498,635 & 26 & Thr & 4 & 442,230 & 416,251 & 397,209 \\
\hline 3490,645 & 3518,640 & 3569,673 & 27 & Ala & 3 & 341,182 & 315,203 & 296,161 \\
\hline 3587,698 & 3615,693 & 3666,725 & 28 & Pro & 2 & 270,145 & 244,166 & 225,124 \\
\hline 3715,793 & 3743,788 & 3794,820 & 29 & Lys & 1 & 173,093 & 147,113 & 128,071 \\
\hline
\end{tabular}

Figure S3. MS analysis of peptide labeled with 1, derived from clone A.17. (A) Direct MS analysis of the peptide absorbed on monomeric avidin resin from a tryptic hydrolysate of A.17 reacted with $\mathbf{1}$. (B) MS/MS analysis of this peptide. (C) Table of theoretical monoisotopic masses of MS/MS fragmentation of VTISC acetamide $_{\text {SGSSSNIGNNYVSWY }}$ phosQQLPGTAPK. Masses found in the MS/MS spectra are in bold with yellow highlight. 


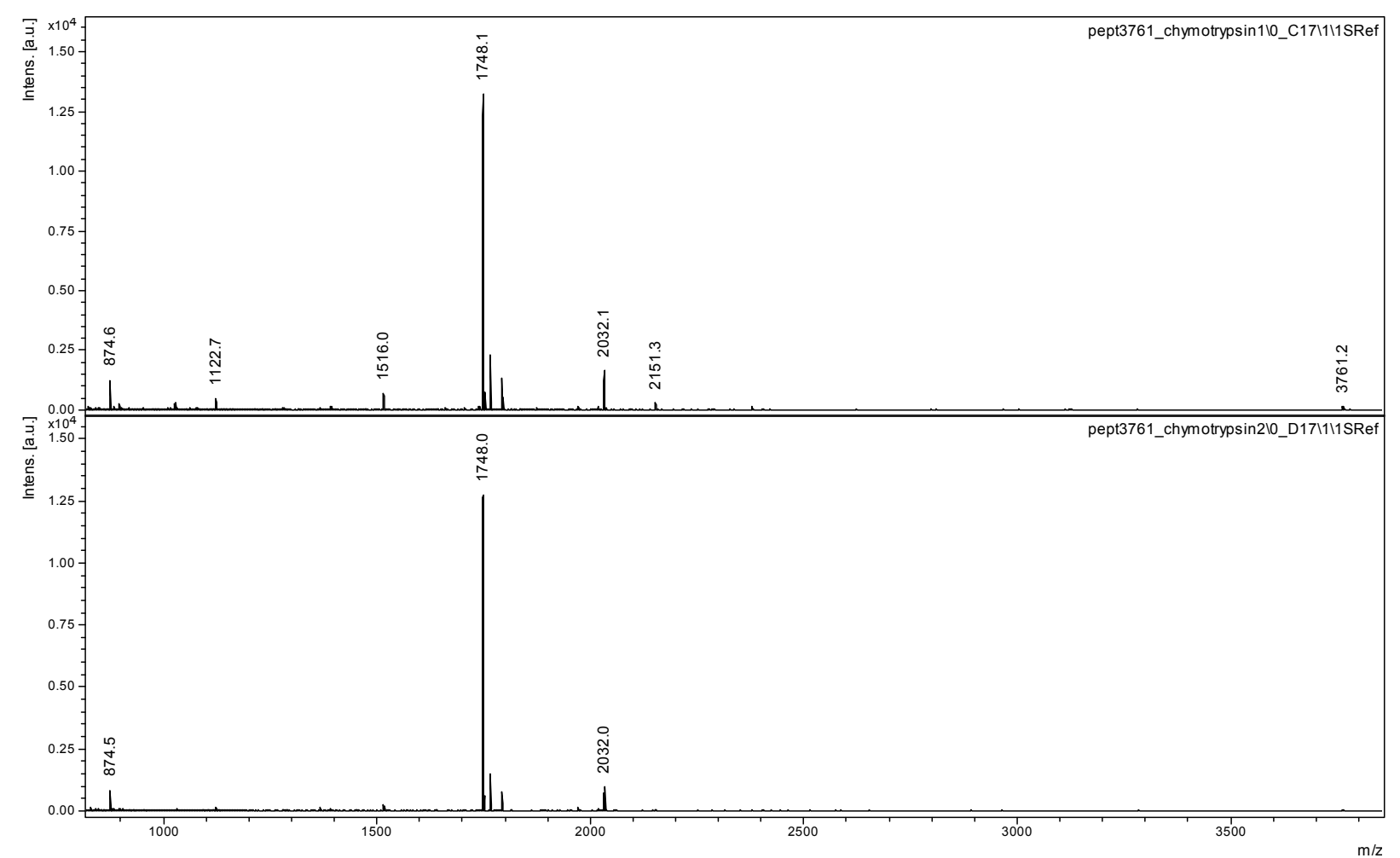

Figure S4. MALDI MS analysis of VL 18-45 (Kabat numbering) peptide digested with chymotrypsin. Two parts were observed $\mathrm{m} / \mathrm{z}=2032.0-$ VTISC $_{\text {acetamide }}$ SGSSSNIGNNYVSW and $\mathrm{m} / \mathrm{z}=1748.1-($ YQQLPGTAPK+ phosphonate $)$. 

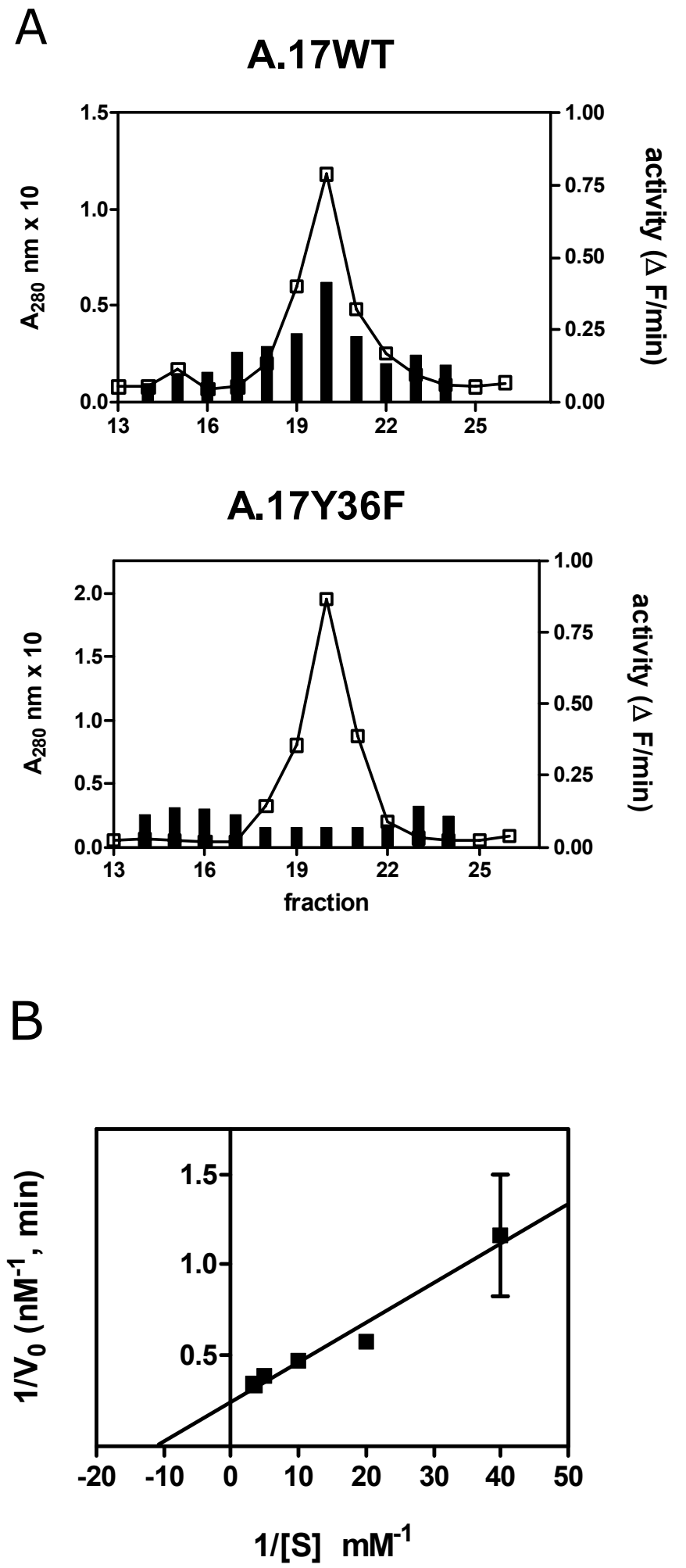

Figure S5. (A) A280 absorbance ( $\square$ ) and superimposed reactivity of FPLC fractions (bars) as determined by F-mca fluorescence changes of scFv A.17 and A.17Y36F. (B) Lineweaver-Burke plot of initial rates of F-mca hydrolysis in presence of $50 \mu \mathrm{g} / \mathrm{ml} \mathrm{A.17.}$ 

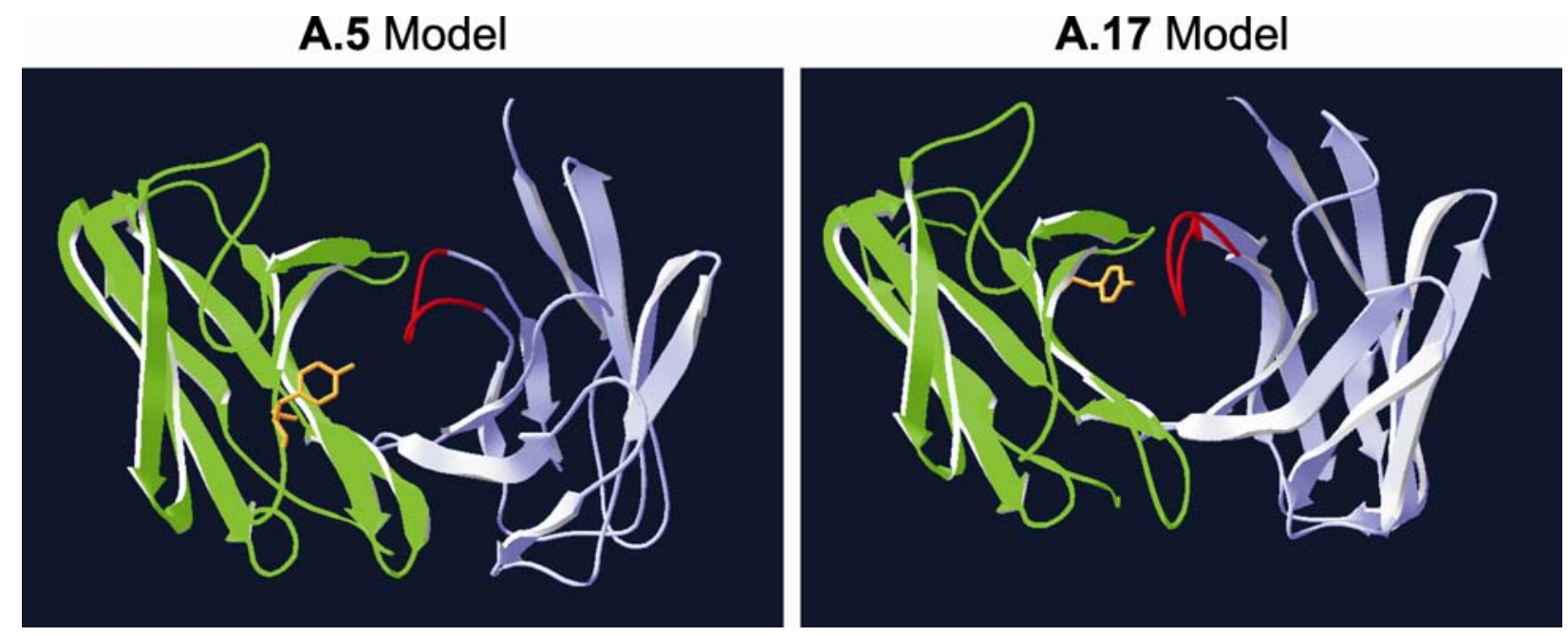

Figure S6. 3D modeling of reactive clones A.5 and A.17. Preliminary models were derived from the three-dimensional structure of homologous antibody molecules (Protein Data Bank: 1F3R-B for VH and 2B0S-L for VL). Side chain replacements and relaxed backbone coordinates obtained by molecular dynamics provided the ternary structures for comparison of sites comprising potential Tyr nucleophiles. Light chains are colored green and heavy chains light blue. Active site Tyr residues are shown in yellow and CDR-H3 in red. 